\title{
Proposição de metodologia para análise de risco em estruturas de pavimentos asfálticos flexíveis
}

\author{
Santi Ferri ${ }^{1}$, Carlos Yukio Suzuki², Felipe Issa Kabbach $\mathrm{Jr}^{3}$, Caio Rubens Santos ${ }^{4}$ e Flaviane Melo Lopes \\ Vallejo 5
}

\begin{abstract}
Resumo: A concepção de empreendimentos de infraestrutura viária envolve diversas fases de planejamento, estudos e projetos. Nestas fases normalmente são discutidas e definidas as possíveis soluções de engenharia a serem aplicadas em cada etapa executiva. Para que as soluções definidas nas fases de planejamento e projeto tenham validade, faz-se necessário garantir que os recursos utilizados, como materiais, mão de obra, máquinas, equipamentos e processos executivos empregados no campo atendam aos pré-requisitos técnicos especificados em projeto. Ocorre, porém, que no Brasil e em outros países do mundo, muitos dos métodos empregados para o dimensionamento de estruturas de pavimentos asfálticos são de natureza determinística, ou seja, utiliza-se de parâmetros médios para caracterização mecânica da estrutura não se preocupando em quantificar os prejuízos ocasionados pela variação de parâmetros proveniente do processo executivo. Na prática, como todo processo industrial, os processos construtivos estão sujeitos a apresentar variações que devem sempre ser aferidas e controladas para garantir a qualidade do produto final. Dentro deste contexto, o presente trabalho apresenta uma metodologia desenvolvida para realizar análises de risco de estruturas de pavimentos asfálticos flexíveis com base em critérios mecanicistas correntemente utilizados no Brasil. Com base nos estudos de caso realizados, verificou-se que a metodologia proposta apresenta boa aplicação prática tanto nas fases de projeto quanto nas fases de controle de qualidade de camadas de infraestrutura de pavimentos recém-executadas.
\end{abstract}

Palavras-chave: análise de risco, controle estatístico, pavimentos asfálticos flexíveis.

\begin{abstract}
The Highway Design Project involve various stages of planning, studies and projects. These phases are usually discussed and defined the possible engineering solutions to be applied in each step of executive phase. To validate the planning and design solutions, it is necessary to ensure that the resources used, such as materials, labor, machinery, equipment and executives processes employed in the field to meet pre-specified technical requirements for the project. Occurs, however, that in Brazil and other countries of the world, many of the methods used for the design of pavements structures are deterministic in nature, in other words, they use the average parameters for mechanical structure characterization not bothering to quantify the damage caused by the variation of parameters from the executive process. In practice, as all industrial processes, construction processes are subject to vary that should always be measured and controlled to ensure product quality. Within this context, this work presents a methodology to carry out risk analysis of flexible pavements structures based on mechanistic criteria commonly used in Brazil. Based on the case studies it was found that the proposed methodology provides good practical application in both project design and quality control phases for new pavement structures.
\end{abstract}

Keywords: risk analysis, statistical control, flexible pavements.

\section{INTRODUÇÃO}

Atualmente, os métodos de dimensionamento de pavimentos correntes no Brasil e em outros países do mundo preconizam a aplicação de fundamentos da Lei de Hooke generalizada para o dimensionamento e/ou a verificação das estruturas de pavimentos. Isto significa que cada material deve ser caracterizado do ponto de vista de seu comportamento mecânico dentro do conjunto estrutural. Para isto, grande parte destes métodos preconiza a adoção de parâmetros médios para o dimensionamento estrutural.

\footnotetext{
1 Santi Ferri, Departamento de Engenharia de Transportes, EP-USP. (santiferri2002@yahoo.com.br)

2 Carlos Yukio Suzuki, Departamento de Engenharia de Transportes, EPUSP. (suzuki@planservi.com.br)

${ }^{3}$ Felipe Issa Kabbach Jr, Departamento de Engenharia de Transportes, EP-USP. (fkabbach@planservi.com.br)

${ }^{4}$ Caio Rubens Santos, Departamento de Engenharia de Transportes,

FIAM-FAAM. (caiorubens@planservi.com.br)

5 Flaviane Melo Lopes Vallejo, Departamento de Engenharia de

Transportes, EP-USP. (flaviane@planservi.com.br)
}

Manuscrito recebido em 15/12/2015 e aprovado para publicação em 29/04/2016

Este artigo é parte de TRANSPORTES v. 25, n. 1, 2017. ISSN: 2237-1346 (online). DOI:10.14295/transportes.v25i1.1049
No Brasil, em especial, o método de dimensionamento utilizado durante muito tempo e um dos mais empregados em projeto ainda é o método puramente empírico, que utiliza o critério de resistência baseado no CBR de camadas granulares. Os valores obtidos no ensaio de CBR não têm relação direta com o estudo da fadiga de misturas asfálticas, que é um dos principais mecanismos de danos em pavimentos nacionais e nem mesmo conseguem captar os benefícios da utilização de solos tropicais (lateríticos) em subleitos e demais camadas dos pavimentos.

O que ocorre na prática é que, como todo processo industrial, os processos construtivos estão sujeitos a variações das mais diversas ordens. Este "fato" exige da comunidade técnica a utilização de critérios rigorosos de controle de qualidade para assegurar a qualidade do produto final.

Para assegurar a qualidade dos materiais no campo, os órgãos rodoviários normalmente desenvolvem especificações técnicas de materiais e serviços para controle da qualidade. As especificações técnicas nacionais vigentes preconizam para cada tipo de material, uma série de exigências técnicas, inclusive para o controle da qualidade da sua execução. Ainda assim, algumas perguntas sempre saltam à vista dos técnicos envolvidos, como por exemplo:

- A especificação de serviço que está-se utilizando para determinado material preconiza que este poderá 
apresentar variações de espessura de até $10 \%$ para mais ou para menos. Esta variação poderá representar riscos de falha precoce na estrutura projetada?

- A especificação de serviço que está-se utilizando para o subleito preconiza um controle deflectométrico no qual permite a ocorrência de até $20 \%$ dos pontos amostrados ultrapassando o valor estabelecido em projeto. Esta variação poderá representar risco de falha precoce na estrutura projetada?

- Se existem riscos, podem ser considerados altos? Existem meios de minimizá-los?

Dentro deste contexto, pode-se afirmar que grande parte dos procedimentos de controle de qualidade utilizados atualmente em obras de pavimentação no Brasil não apresentam muitas alternativas viáveis para a tomada de ações corretivas. Isto muitas vezes impede que o controle seja eficiente, pois, na grande maioria dos casos a única ação corretiva indicada é a reconstrução integral do pavimento dito "deficiente", independentemente do "grau de deficiência". Isto poderia implicar em comprometimento de grande parte dos recursos físicos e financeiros alocados, podendo inclusive ocasionar atrasos no andamento das obras e refletindo em prejuízos econômicos e sociais. Além disso, muitas das metodologias empregadas atualmente não se preocupam em quantificar os eventuais prejuízos ocasionados em "execuções deficientes" de estruturas de pavimentos rodoviários, nem mesmo em propor alternativas para a tomada de ações corretivas.

Dentro deste contexto, Andreatini (1988) propôs a aplicação de metodologias de controle estatístico específicos para a área de pavimentação e baliza as principais metodologias de controle encontradas atualmente nas normas e especificações de diversos órgãos rodoviários nacionais tais como DNIT, DER/SP, DERSA, PMSP/SIURB etc. A publicação FHWA-HRT-04-046 (2004) apresenta uma ampla revisão acerca dos índices a serem utilizados para fins de controle estatístico da qualidade em obras viárias. O relatório NCHRP 10-65 do TRB (2009) apresenta uma ampla revisão acerca dos dispositivos e equipamentos utilizados para controle dos materiais no campo. Entre outras pesquisas relevantes na área de controle de qualidade de obras de infraestrutura viária podem se citar as pesquisas de McCabe et al. (1999), Pathomvanich (2002), Apeagyei \& Hossain (2010), Gharaibeh et al. (2010).

O objetivo do presente trabalho é propor ferramentas que permitirão inferir o grau de risco de falha da estrutura recém-projetada, frente às especificações técnicas de projeto, às condições executivas e com base em dados obtidos diretamente do controle deflectométrico realizado no campo.

As sugestões apresentadas no presente trabalho contemplam a utilização de 3 tipos de deflectômetros usualmente empregados no país, quais sejam: a Viga Benkelman, o Falling Weight Deflectometer (FWD) e o Light Weight Deflectometer (LWD).

\section{DESENVOLVIMENTO DE METODOLOGIA DE ANÁLISE DE RISCO}

A metodologia idealizada pode ser sintetizada em oito etapas:
- ETAPA A: Coleta de informações de projeto (número N, parâmetros estruturais do pavimento);

- ETAPA B: Adoção de faixas de variações para os parâmetros estruturais do pavimento;

- ETAPA C: Simulações de casos das combinações possíveis (técnica de Monte Carlo);

- ETAPA D: Determinação das respostas estruturais do pavimento em cada caso simulado;

- ETAPA E: Determinação do número $\mathrm{N}$ admissível em cada caso simulado;

- ETAPA F: Análise probabilística dos casos simulados;

- ETAPA G: Comparação com os valores de projeto;

- ETAPA H: Embasamento para tomada de ações corretivas.

A seguir será detalhada cada uma das etapas propos-

tas.

\subsection{Etapa A: Coleta de informações de projeto}

Esta etapa consiste em coletar as informações de tráfego e da estrutura do pavimento tal como previsto em projeto. O tráfego deverá ser admitido em termos de Número $\mathrm{N}$ acumulado para o horizonte de projeto. A metodologia, tal como proposta no presente trabalho, não se aplica a análise de dano acumulado.

Importante citar que a aplicação da metodologia de risco proposta no presente trabalho independe da metodologia aplicada para cálculo de fatores de equivalência de carga e cálculo de número $\mathrm{N}$ adotado no projeto, sendo utilizado o valor de número $\mathrm{N}$ de projeto apenas como uma referência para as análises de risco relativo. No presente estudo de caso foi utilizada a metodologia proposta na instrução de projeto IP-DE-P00/001 do DER/SP que considera a determinação dos números $\mathrm{N}$ acumulados pelas metodologias da USACE e AASHTO/93 para aplicação nos modelos de fadiga da norma citada, respeitando o critério normativo vigente. Os autores reconhecem que há uma série de restrições na adoção destas metodologias para determinação do número $\mathrm{N}$ para fins de análise mecanicista, porém reitera que a alteração da metodologia de cálculo do número $\mathrm{N}$ de projeto não representaria prejuízo algum à aplicação da metodologia proposta no presente trabalho, podendo ser facilmente adaptada.

Já para caracterização da estrutura do pavimento, deverão ser coletadas as informações do tipo de pavimento adotado, os materiais, suas respectivas espessuras e módulos, e os valores de controle deflectométrico para cada camada.

\subsection{Etapa B: Adoção de faixas de variações para os parâmetros estruturais do pavimento}

A etapa $B$ consiste em determinar faixas de variações para os parâmetros estruturais do pavimento. $\mathrm{O}$ procedimento pode ser realizado basicamente de duas formas:

a. $\mathrm{Na}$ fase de projeto

Adotando-se as faixas de variação admitida para os parâmetros, conforme preconizado nas especificações de serviço do órgão competente ou conforme definido no próprio projeto.

b. Na fase de controle de qualidade da obra 
Tabela 1. Valores para o coeficiente $\alpha$ em função do tipo de equipamento utilizado e do parâmetro de controle utilizado nos ensaios de campo

\begin{tabular}{|c|c|c|c|c|c|c|c|c|}
\hline \multirow[b]{2}{*}{ Tipo de Ensaio } & \multirow[b]{2}{*}{$\begin{array}{c}\text { Padrão de } \\
\text { carregamento }\end{array}$} & \multicolumn{7}{|c|}{ Valores de $\alpha$ em função do Parâmetro de Curvatura (PC) } \\
\hline & & $\mathrm{D}_{0}$ & $\mathrm{D}_{25}$ & $\mathrm{D}_{120}$ & $\begin{array}{c}\mathrm{SCI}= \\
\mathrm{D}_{0}-\mathrm{D}_{30}\end{array}$ & $\begin{array}{c}\mathrm{CF}= \\
\mathrm{D}_{0}-\mathrm{D}_{20}\end{array}$ & $\begin{array}{c}\mathrm{BDI}= \\
\mathrm{D}_{30}-\mathrm{D}_{60}\end{array}$ & $\begin{array}{c}\mathrm{BCI}= \\
\mathrm{D}_{60}-\mathrm{D}_{90}\end{array}$ \\
\hline \multirow{2}{*}{ Viga Benkelman } & $\begin{array}{c}\text { Usual em projeto } \\
(\mathrm{p}=0,56 \mathrm{MPa})\end{array}$ & 7.895 & 3.678 & 857 & 4.726 & 3.566 & 1.479 & 538 \\
\hline & $\begin{array}{l}\text { PMSP/SIURB IP-08 } \\
\quad(p=0,60 \mathrm{MPa})\end{array}$ & 6.457 & 3.492 & 853 & 3.398 & 2.406 & 1.390 & 524 \\
\hline FWD & $\mathrm{P}=4.100 \mathrm{kgf} ; \mathrm{r}=0,15 \mathrm{~m}$ & 13.875 & 4.379 & 874 & 10.287 & 8.191 & 1.843 & 589 \\
\hline \multirow{3}{*}{ LWD } & $\mathrm{P}=707 \mathrm{kgf} ; \mathrm{r}=0,15 \mathrm{~m}$ & 2.393 & 755 & 151 & 1.774 & 1.412 & 341 & 79 \\
\hline & $\mathrm{P}=1.060 \mathrm{kgf} ; \mathrm{r}=0,15 \mathrm{~m}$ & 3.587 & 1.132 & 226 & 2.660 & 2.118 & 477 & 152 \\
\hline & $\mathrm{P}=1.000 \mathrm{kgf} ; \mathrm{r}=0,15 \mathrm{~m}$ & 3.384 & 1.068 & 213 & 2.509 & 1.998 & 482 & 111 \\
\hline
\end{tabular}

Medindo-se as variações de parâmetros estruturais (espessuras e módulos) ocorridas na fase construtiva.

Os módulos poderão ser inferidos através de ensaios de módulo de resiliência realizados em laboratório a partir de amostras extraídas do campo e/ou através da retroanálise dos valores de deflexão obtidos no campo. O ideal é que ambos os ensaios sejam realizados.

Para obtenção do valor de módulo da camada de infraestrutura a partir dos valores de deflexão ou de parâmetros de curvatura da bacia de deflexões, pode-se fazer uso das equações (1) e (2) indicadas a seguir.

$$
E s l=\frac{\alpha}{P C}
$$

Onde: $E s l=$ módulo elástico do subleito $(\mathrm{MPa}) ; \alpha=$ coeficiente de regressão (N/dm), dado segundo a Tabela 1 ; $P C=$ parâmetro de curvatura obtido no ensaio deflectométrico $-D_{0}, D_{25}, D_{120}, S C I$ (Índice da Curvatura da Superfície), $C F$ (Fator de Curvatura), BDI (Índice de Dados a Base) ou $B C I$ (Índice da Curvatura da Base) em $10^{-5} \mathrm{~m}$ ou $10^{-}$ ${ }^{2} \mathrm{~mm}$.

Onde: $\mathrm{D}_{0}, \mathrm{D}_{20}, \mathrm{D}_{25}, \mathrm{D}_{30}, \mathrm{D}_{60}, \mathrm{D}_{90}$ e $\mathrm{D}_{120}$ são deflexões afastadas a $0,20,25,30,60,90$ e $120 \mathrm{~cm}$ do ponto de aplicação da carga em $10^{-5} \mathrm{~m}$ ou $10^{-2} \mathrm{~mm}$.

No caso do parâmetro Raio de curvatura, a estimativa do módulo pode ser realizada através da equação a seguir e dos fatores indicados na Tabela 2:

$$
E s l=\beta \times R c
$$

Onde:

$E s l=$ módulo elástico do subleito $(\mathrm{MPa}) ; \beta=$ coeficiente de regressão $\left(\mathrm{N} / \mathrm{m}^{3}\right)$, dado segundo a Tabela $2 ; \mathrm{Rc}=$ Raio de curvatura em metros: $\left[R c=3125 /\left(D_{0}-D_{25}\right)\right]$, conforme DNIT.

Tabela 2. Valores para o coeficiente $\beta$ para retroanálise do valor de módulo a partir de $R c$

\begin{tabular}{ccc}
\hline $\begin{array}{c}\text { Tipo de } \\
\text { Ensaio }\end{array}$ & Padrão de Carregamento & Coeficiente $\boldsymbol{\beta}$ \\
\hline $\begin{array}{c}\text { Viga } \\
\text { Benkelman }\end{array}$ & $\begin{array}{c}\text { Usual em projetos ( } \mathrm{p}=0,56 \mathrm{MPa}) \\
\text { PMSP/SIURB IP-08 } \\
(\mathrm{p}=0,60 \mathrm{MPa})\end{array}$ & 1,35 \\
\hline FWD & $\mathrm{P}=4.100 \mathrm{kgf} ; \mathrm{r}=15 \mathrm{~cm}$ & 0,95 \\
\hline \multirow{2}{*}{ LWD } & $\mathrm{P}=707 \mathrm{kgf} ; \mathrm{r}=15 \mathrm{~cm}$ & 30,4 \\
& $\mathrm{P}=1.060 \mathrm{kgf} ; \mathrm{r}=15 \mathrm{~cm}$ & 0,52 \\
& $\mathrm{P}=1.000 \mathrm{kgf} ; \mathrm{r}=15 \mathrm{~cm}$ & 0,79 \\
\hline
\end{tabular}

A equação (1) e a equação (2) foram obtidas através de simulações teóricas realizadas pelos autores com o uso do software Elsym5.

As equações (1) e (2) devem ser aplicadas para cada um dos dados obtidos diretamente do controle deflectométrico de campo (deflexão máxima e/ou demais parâmetros deflectométricos). Por exemplo, no caso do controle deflectométrico contemplar apenas o valor de $\mathrm{D}_{0}$ - deflexão máxima, os valores de $\mathrm{D}_{0}$ são substituídos diretamente no valor de PC da equação, e são então aplicados o valor de alfa relativo ao tipo de ensaio. Desta maneira, por retroanálise, serão inferidos os valores de módulo do subleito em cada ponto do ensaio. Após isto, para um determinado segmento de controle, verificam-se os parâmetros estatísticos dos valores de módulo retroanalisados (média, desvio padrão, valores máximos e mínimos etc.).

\subsection{Etapa C: Simulações de casos das combinações possíveis (técnica de Monte Carlo)}

Esta etapa consiste em combinar, aleatoriamente e dentro das faixas de variações admitidas na etapa B, os valores dos parâmetros estruturais do pavimento em termos de módulos e espessuras das camadas.

Quanto maior o número de casos simulados, melhor a confiabilidade e menores taxas de "erro" na interpretação dos dados. O "erro" pode ser calculado pelas equações indicadas a seguir.

$$
\varepsilon=\frac{3 s}{\sqrt{N i}}
$$

$$
\varepsilon \%=\frac{\varepsilon}{\bar{x}}
$$

Onde: $\varepsilon \%=$ erro relativo; $\varepsilon=$ erro total; $\mathrm{s}=$ desvio padrão; $N i=$ número de iterações; $\bar{x}=$ média.

No presente trabalho, esta etapa foi realizada a partir de planilha eletrônica desenvolvida em Excel®, através do uso da função “ALEATÓRIOENTRE (inferior;superior)".

\subsection{Etapa D: Determinação das respostas estruturais do pavimento em cada caso simulado}

A etapa D consiste em inferir as respostas estruturais dos diversos casos simulados na etapa anterior quando da aplicação da carga similar à do eixo padrão rodoviário. 
Tabela 3. Modelos propostos para cálculo das respostas estruturais

\begin{tabular}{ccc}
\hline Tipo de Pavimento & Modelo & Equação \\
\hline Flexível & $\varepsilon_{t}=a+b \cdot H_{2}+c \cdot H_{3}+d \cdot E_{1}+e \cdot E_{2}+f \cdot E_{3}$ \\
& $D_{F W D}=10^{g} \cdot H_{2}{ }^{h} \cdot H_{3}{ }^{i} \cdot E_{1}{ }^{j} \cdot E_{2}{ }^{k} \cdot E_{3}{ }^{l}$
\end{tabular}

As respostas estruturais devem ser coletadas em termos de deslocamentos recuperáveis (deflexão) e deformações específicas nos pontos críticos das estruturas de pavimento.

No caso do presente trabalho, a exemplo do que foi apresentado na instrução de projeto IP-08 (PMSP/SIURB, 2004), foram realizadas simulações com o software Elsym5, para o caso de sistemas de três camadas (pavimentos flexíveis), para obtenção de modelos matemáticos que possibilitem o cômputo das respostas estruturais do pavimento de forma rápida e prática. Os modelos obtidos são apresentados na Tabela 3. As constantes de regressão dos modelos propostos são apresentadas na Tabela 4. As variações de parâmetros admitidas para realização deste estudo teórico são apresentadas na Tabela 5 .

As letras de $\boldsymbol{a}$ até $\boldsymbol{l}$ da Tabela 3 representam as constantes de regressão obtidas no estudo, cujos valores são apresentados na Tabela 4 seguir.

Tabela 4. Constantes de regressão dos modelos propostos

\begin{tabular}{cccc}
\hline & Eq. (5) & & Eq. (6) \\
\hline$a$ & 7,52927 & $g$ & 4,88944 \\
$b$ & $-3,0500$ & $h$ & $-0,12339$ \\
$c$ & $-13,8120$ & $i$ & $-0,59719$ \\
$d$ & $-0,00344$ & $j$ & $-0,59105$ \\
$e$ & $-0,00506$ & $k$ & $-0,18561$ \\
$f$ & $-0,00031$ & $l$ & $-0,22453$ \\
$R^{2}$ & 0,91 & $R^{2}$ & 0,99 \\
\hline
\end{tabular}

Onde: $\varepsilon t=$ deformação horizontal de tração na fibra inferior da camada de revestimento $\left(10^{-4} \mathrm{~m} / \mathrm{m}\right)$; $D_{F W D}=$ deflexão na superfície do sistema de três camadas nas condições do ensaio com FWD $(0,01 \mathrm{~mm}) ; H_{2}=$ espessura da camada de base $(\mathrm{m}) ; H_{3}=$ espessura da camada de revestimento $(\mathrm{m}) ; E_{l}=$ módulo elástico da camada de subleito ou infraestrutura equivalente $(\mathrm{MPa}) ; E_{2}=$ módulo elástico da camada de base granular $(\mathrm{MPa})$ - valor mínimo de 200MPa e máximo de $300 \mathrm{MPa} ; E_{3}=$ módulo elástico da camada de revestimento asfáltico $(\mathrm{MPa})$ - valor mínimo de
2.000MPa e máximo de $5.000 \mathrm{MPa} ; \mu 1, \mu 2$ e $\mu 3=$ coeficientes de Poison das camadas de subleito, base granular e revestimento asfáltico respectivamente (adimensionais). Nota: $1 \mathrm{MPa} \sim 10 \mathrm{kgf} / \mathrm{cm}^{2}$.

$\mathrm{O}$ presente procedimento parte do princípio que todos os materiais a serem utilizados no projeto sejam previamente caracterizados em termos de seu comportamento mecânico, especialmente em termos de seus valores de módulo. Uma vez obtidos os valores de módulo de laboratório, recomenda-se que estes sejam comparados com os valores máximos e mínimos apresentados na Tabela 5 para cada tipo de material - infraestrutura, base e revestimento. Caso os materiais apresentem valores de módulo sensivelmente inferiores ou superiores aos valores de módulos utilizados nas simulações (Tabela 5), não se recomenda o uso das equações estabelecidas na Tabela 3 , sendo mais adequado, nestes casos, o estabelecimento de novas equações de regressão a partir dos valores obtidos em laboratório.

\subsection{Etapa E: Determinação do número $\mathrm{N}$ admissível em cada caso simulado}

Na etapa E propõe-se a aplicação dos modelos de fadiga tais como preconizam os métodos nacionais de dimensionamento (DER/SP, PMSP/SIURB e DNIT) com o objetivo de inferir o número de ciclos (número $\mathrm{N}$ ) admissível, para cada um dos casos simulados nas etapas anteriores.

No caso do presente trabalho, foram utilizados os modelos de fadiga indicados na Tabela 6 a seguir.

Os modelos apresentados na Tabela 6 foram utilizados no presente estudo apenas como exemplo de aplicação da metodologia proposta. Outros modelos, inclusive aqueles ajustados para pavimentos em serviço em regiões tropicais podem ser utilizados sem prejuízos à metodologia proposta, apenas observando a compatibilidade com as normas aplicáveis e/ou à metodologia de cálculo do número $\mathrm{N}$.

Tabela 5. Parâmetros utilizados para a obtenção dos modelos de regressão

\begin{tabular}{|c|c|c|c|c|c|c|c|c|c|c|}
\hline $\begin{array}{c}\text { Tipo de } \\
\text { Pavimento }\end{array}$ & Equaçõos & $\begin{array}{c}\text { No. de casos } \\
\text { simulados (Elsym-5) }\end{array}$ & $\begin{array}{l}\mathbf{H}_{2} \\
(\mathbf{m})\end{array}$ & $\begin{array}{l}\mathbf{H}_{3} \\
(\mathbf{m})\end{array}$ & $\begin{array}{c}\mathbf{E}_{1} \\
(\mathbf{M P a})\end{array}$ & $\begin{array}{c}\mathbf{E}_{2} \\
(\mathbf{M P a})\end{array}$ & $\begin{array}{c}\mathbf{E}_{3} \\
(\mathbf{M P a})\end{array}$ & $\mu 1$ & $\mu 2$ & $\mu 3$ \\
\hline Flexível & $(5)$ e (6) & 1.575 & $\begin{array}{l}0,10 \\
0,12 \\
0,15 \\
0,17 \\
0,20\end{array}$ & $\begin{array}{l}0,05 \\
0,07 \\
0,10 \\
0,12 \\
0,15 \\
0,17 \\
0,20\end{array}$ & $\begin{array}{c}50 \\
70 \\
100 \\
150 \\
200\end{array}$ & $\begin{array}{l}200 \\
250 \\
300\end{array}$ & $\begin{array}{l}2.000 \\
3.500 \\
5.000\end{array}$ & 0,45 & 0,40 & 0,35 \\
\hline
\end{tabular}

Tabela 6. Modelos de fadiga utilizados

\begin{tabular}{cccccc}
\hline Parâmetro & Símbolo & Material & Norma Aplicável & Modelo & Equação \\
\hline $\begin{array}{c}\text { Deslocamento vertical na } \\
\text { superfície do pavimento }\end{array}$ & $\mathrm{D}_{0}$ & (Revestimento) & $\begin{array}{c}\text { DNER } \\
\text { PRO-269/94 }\end{array}$ & $N_{U S A C E}=5,548 \times 10^{16} \times\left(\frac{1}{D_{0}}\right)^{5,319}$ & $(7)$ \\
\hline $\begin{array}{c}\text { Deformação horizontal de } \\
\text { tração na fibra inferior do } \\
\text { revestimento }\end{array}$ & $\varepsilon \mathrm{t}$ & CBUQ & $\begin{array}{c}\text { DER/SP } \\
\text { IP-DE-P00/001 (2006) }\end{array}$ & $N_{\text {AASHTO }}=1,092 \times 10^{-6} \times\left(\frac{1}{\varepsilon_{t}}\right)^{3,512}$ & $(8)$ \\
\hline
\end{tabular}




\subsection{Etapa F: Análise probabilística dos casos simulados}

Esta etapa consiste na montagem de curvas de densidade (curva de Gauss) e de densidade acumulada (curva de probabilidades) com os valores de número $\mathrm{N}$ admissíveis obtidos para cada caso simulado nas etapas anteriores.

\subsection{Etapa G: Comparação com os valores de projeto}

Esta etapa consiste em confrontar os valores de número $\mathrm{N}$ (atuante) admitido em projeto para o horizonte de projeto selecionado, com a curva de densidade acumulada obtida na etapa F. Pretende-se com isto inferior o nível de risco da estrutura que apresentar desempenho inferior àquele admitido em projeto, com base nas faixas de variações admitidas para os parâmetros estruturais na etapa construtiva.

Nesta etapa, há que se considerarem os conceitos de "risco" e de "confiabilidade" de projeto.

Segundo o guia do dimensionamento da AASHTO/93, "Confiabilidade é a probabilidade de um tipo particular de "falha" (ou combinação de manifestações de falhas) permanecer abaixo ou dentro dos limites admissíveis durante o período de projeto".

O guia da AASHTO/93 também cita outra definição mais ligada a projeto:

"A Confiabilidade de um procedimento de projeto baseado em desempenho é a probabilidade de uma estrutura de pavimento projetada obter desempenho satisfatório sob as condições de tráfego e ambientais durante o período de projeto selecionado".

O conceito de confiabilidade é bastante importante para balizar as decisões neste tipo de análise probabilística. Obras de grande porte demandam investimentos maiores e, portanto, a baixa confiabilidade nestes casos, pode representar prejuízos elevados, enquanto em investimentos de menor porte, pode-se exigir um nível de confiabilidade um pouco inferior. O guia da AASHTO de 1993 contém algumas diretrizes para seleção de níveis de confiabilidade a serem adotados em projeto, que podem balizar as análises desta etapa. A Tabela 7 ilustra os níveis de confiabilidade sugeridos pela AASHTO/93, em função da classificação funcional da via. O guia também sugere uma metodologia para obtenção do nível ótimo de confiabilidade, através da estimativa dos custos iniciais e futuros envolvidos com a construção e manutenção da via. No presente trabalho, as análises serão balizadas pelas recomendações contidas na Tabela 7.

Tabela 7. Níveis de Confiabilidade sugeridos para várias classificações funcionais de vias (AASHTO/1993)

\begin{tabular}{lcc}
\hline \multirow{2}{*}{ Classificação das Vias } & \multicolumn{2}{c}{ Confiabilidade } \\
\cline { 2 - 3 } & Urbana & Rural \\
\hline Interestadual & $85-99,9$ & $80-99,9$ \\
Vias arteriais principais & $80-99$ & $75-95$ \\
Vias coletoras & $80-95$ & $75-95$ \\
Vias locais & $50-80$ & $50-80$ \\
\hline
\end{tabular}

\subsection{Etapa H: Embasamento para tomada de ações corretivas}

A etapa $\mathrm{H}$ consiste em obter respostas rápidas de como proceder no caso de tomada de ações corretivas com o objetivo de elevar os níveis de confiabilidade relativa ao projeto da estrutura de pavimento e consequentemente minimizarem-se os riscos de "falha". Este procedimento pode ser realizado basicamente de duas formas:

a) Executando novamente as etapas de A até G, supondo-se melhorias na estrutura do pavimento (incrementar espessuras das camadas, elevar resistência dos materiais, melhorar o módulo do subleito etc.).

b) Realizando a regressão estatística dos valores obtidos nas simulações e realizando-se melhorias na estrutura, conforme citado no item anterior.

No caso desta última opção, propõe-se a realização de procedimento de regressão estatística dos valores obtidos no estudo, com a finalidade de se obter uma equação que expresse, para cada caso particular, a Probabilidade de sucesso (P) em função da alteração dos parâmetros estruturais do pavimento.

A obtenção da equação de regressão permite, na etapa de obra, o embasamento necessário para a tomada de ações corretivas rápidas, após o controle da camada subjacente. Por exemplo, uma vez construída e controlada a camada de subleito, obtém-se o valor médio de módulo retroanalisado aquém do valor mínimo de projeto. Utilizando-se a equação, pode-se propor melhoria nas camadas de base ou revestimento, e computarem-se os novos valores de Probabilidade de sucesso, até que estes atinjam valores ideais. Exemplos serão apresentados nos estudos de caso do presente trabalho.

\section{ESTUDO DE CASO - PAVIMENTO FLEXÍVEL}

\subsection{Etapa A - Coleta de informações de projeto}

Este estudo foi conduzido utilizando-se os dados de projeto e de controle deflectométrico da obra de duplicação de uma rodovia do interior do estado de São Paulo. O objetivo do estudo foi o realizar a análise de risco proposta no presente trabalho. Os parâmetros estruturais do pavimento admitidos em projeto são apresentados na Tabela 8 .

Para analogia ao modelo proposto na etapa D (item 2.4) deste trabalho, utilizaram-se, para análise, os dados de deflexão obtidos na superfície da camada de reforço do subleito. Deste modo, esta camada configurará, nas análises, a camada de infraestrutura equivalente que dará suporte ao pavimento flexível Figura 1.

O Número $\mathrm{N}$ projetado para o horizonte de 10 anos foi de $1,78 \times 10^{7}$ pela metodologia da USACE e de $5,92 \times 10^{6}$ pela metodologia da AASHTO/93.

Tabela 8. Estrutura indicada no projeto

\begin{tabular}{cccccc}
\hline Camada & Material & Espessura $(\mathrm{cm})$ & CBRp (\%) & Módulo (MPa) & $\begin{array}{c}\text { Deflexão admissível [VB] } \\
\left(10^{-2} \mathrm{~mm}\right)\end{array}$ \\
\hline Revestimento & CBUQ & 10,0 & - & 3.750 & 57 \\
Base & BGS & 20,0 & - & 300 & 74 \\
Reforço & Solo selecionado & 25,0 & 20 & 200 & 91 \\
Subleito & Solo local & - & 8 & 80 & 126 \\
\hline
\end{tabular}


FERRI, S.; SUZUKI, C.Y.; KABBACH, F.I.; SANTOS, C.R.; VALLEJO, F.M.L.

\begin{tabular}{|c|c|c|c|}
\hline \multicolumn{3}{|c|}{ ESTRUTURA DE PROJETO } & \multirow[b]{2}{*}{$\begin{array}{l}\text { (deflexões de } \\
\text { controle } D_{0 p} \text { ) }\end{array}$} \\
\hline $\begin{array}{c}\text { Espessura } \\
\text { (cm) }\end{array}$ & $\begin{array}{l}\text { Módulo } \\
\text { (MPa) }\end{array}$ & Materiais & \\
\hline & & & $7 \times 0,01 \mathrm{~mm}$ \\
\hline 10,0 & 3.750 & CBUQ & $4 \times 0,01 \mathrm{~mm}$ \\
\hline 20,0 & 300 & BGS & $1 \times 0,01 \mathrm{~mm}$ \\
\hline 25,0 & 200 & REFORÇO & $26 \times 0,01 \mathrm{~mm}$ \\
\hline - & 80 & SUBLEITO & \\
\hline
\end{tabular}

Figura 1. Analogia utilizada no estudo

Tabela 9. Estatística dos valores pontuais medidos no segmento homogêneo (infraestrutura equivalente)

\begin{tabular}{|c|c|c|c|c|c|c|c|c|c|c|c|}
\hline $\begin{array}{l}\text { Seg. } \\
\text { núm. }\end{array}$ & $\begin{array}{c}\text { Início } \\
\text { (estaca) }\end{array}$ & $\begin{array}{c}\text { Fim } \\
\text { (estaca) }\end{array}$ & $\begin{array}{l}\text { Ext. } \\
(\mathrm{m})\end{array}$ & $\begin{array}{l}\text { Quant. } \\
\text { pontos }\end{array}$ & $\begin{array}{c}\text { Media } \\
D_{0} \\
(0,01 \mathrm{~mm})\end{array}$ & $\begin{array}{c}\text { Desvio } \\
\text { Padrão } \\
\left(D_{0}\right)\end{array}$ & $\begin{array}{c}\text { CV } \\
\left(D_{0}\right)\end{array}$ & $\begin{array}{l}\text { Media } \\
\text { Eeq, rsl } \\
\text { (MPa) }\end{array}$ & $\begin{array}{c}\text { Desvio } \\
\text { Padrão } \\
(\text { Eeq, rsl })\end{array}$ & $\begin{array}{c}\text { CV } \\
(\text { Eeq, rsl })\end{array}$ & $\begin{array}{c}\text { Variação } \\
\text { máxima de } E e q, r s l \\
\text { em relação à } \\
\text { média }\end{array}$ \\
\hline 1 & 66 & 74 & 160 & 16 & 61,4 & 6,2 & $10,1 \%$ & 129,8 & 13,2 & $10,2 \%$ & $20,8 \%$ \\
\hline
\end{tabular}

\subsection{Etapa B - Adoção de faixas de variações para os parâmetros estruturais do pavimento}

Serão adotadas as seguintes faixas de variações para os parâmetros estruturais do pavimento:

- Variações em espessuras, para os materiais CBUQ e BGS: $10 \%$ (valores admitidos com base nas especificações técnicas de materiais e serviços vigentes do DER/SP);

- Variação nos valores dos módulos dos materiais CBUQ e BGS: 10\% (valores arbitrados nesta análise que poderiam ser aferidos nas etapas construtivas por meio de ensaios de laboratório e/ou ensaios de campo);

- Variação no módulo da infraestrutura equivalente (conjunto subleito + reforço do subleito): $24,6 \%$ (valores estimados com base em procedimentos de controle deflectométrico com viga Benkelman e retroanálise dos valores de módulo, conforme detalhado abaixo).

No caso deste estudo, para a estimativa da variação dos valores de módulo da infraestrutura equivalente, foram utilizados os dados do controle deflectométrico do pavimento em um determinado lote de construção.

A amostragem utilizada no controle foi aquela recomendada pela especificação do DER/SP para subleito e reforço do subleito (ET-DE-P00/001 e ET-DE-P00/002 respectivamente), ou seja, controle com Viga Benkelman, e leituras, no mínimo, a cada $20 \mathrm{~m}$ por faixa alternada ou a cada $40 \mathrm{~m}$ na mesma faixa, com determinação de $\mathrm{D}_{0}$. Neste

caso não foi realizada a segmentação homogênea do lote amostrado, uma vez que o segmento apresenta apenas $160 \mathrm{~m}$ de extensão. A Tabela 9 apresenta as estatísticas dos valores pontuais medidos no topo do sistema de infraestrutura do segmento em análise (conjunto subleito / reforço do subleito). A retroanálise foi realizada a partir dos valores de deflexão máxima $\left(\mathrm{D}_{0}\right)$, com base no uso da equação 1 e os coeficientes para o ensaio de Viga Benkelman indicados na Tabela 1.

O valor de Variação máxima do módulo equivalente (Eeq,rsl) é obtido através do valor máximo da diferença percentual, em módulo, dos valores pontuais e médio no segmento analisado. Este valor será utilizado nas simulações de Monte Carlo para representar a variação limitante a que os valores de módulo estarão sujeitos em cada segmento de análise.

\subsection{Etapas C, D e E}

No presente estudo, as etapas C, D, E foram sintetizadas em uma única planilha, sendo:

- Etapa C: geram-se valores aleatórios dentro dos intervalos de variações definidos na etapa anterior;

- Etapa D: computam-se os valores estimados de deflexão e deformação atuante nos pontos críticos da estrutura (utilizando-se os modelos da Tabela 3);

- Etapa E: aplicam-se os modelos de fadiga (utilizando-se os modelos da Tabela 6).

A Tabela 10 ilustra a realização das etapas descritas acima.

Tabela 10. Planilha contendo as etapas C, D e E

\begin{tabular}{ccccccccccc}
\hline \multicolumn{1}{c}{ Etapa C } & \multicolumn{1}{c}{ Etapa D } & \multicolumn{2}{c}{ Etapa E } \\
\hline Caso & $\begin{array}{c}\mathrm{H}_{1} \\
(\mathrm{~cm})\end{array}$ & $\begin{array}{c}\mathrm{H}_{2} \\
(\mathrm{~cm})\end{array}$ & $\begin{array}{c}\mathrm{H}_{3} \\
(\mathrm{~cm})\end{array}$ & $\begin{array}{c}\mathrm{E}_{1} \\
(\mathrm{MPa})\end{array}$ & $\begin{array}{c}\mathrm{E}_{2} \\
(\mathrm{MPa})\end{array}$ & $\begin{array}{c}\mathrm{E}_{3} \\
(\mathrm{MPa})\end{array}$ & $\begin{array}{c}\varepsilon_{\mathrm{t}} \\
\left(10^{-4} \mathrm{~mm} / \mathrm{mm}\right)\end{array}$ & $\begin{array}{c}\mathrm{D}_{0} \\
(0,01 \mathrm{~mm})\end{array}$ & $\mathrm{Nf}(\varepsilon \mathrm{ct})$ & $\mathrm{Nf}\left(\mathrm{D}_{0}\right)$ \\
\hline 1 & - & 22 & 11 & 87 & 270 & 3.273 & $\mathbf{2 , 6 6}$ & $\mathbf{5 1 , 8 8}$ & $3,87 \mathrm{E}+06$ & $4,17 \mathrm{E}+07$ \\
\hline 2 & - & 20 & 9 & 87 & 241 & 3.245 & $\mathbf{3 , 1 5}$ & $\mathbf{6 0 , 5 6}$ & $2,13 \mathrm{E}+06$ & $1,83 \mathrm{E}+07$ \\
\hline 3 & - & 20 & 11 & 59 & 234 & 3.804 & $\mathbf{2 , 8 3}$ & $\mathbf{6 5 , 5 7}$ & $3,10 \mathrm{E}+06$ & $1,20 \mathrm{E}+07$ \\
\hline 4 & - & 21 & 10 & 83 & 251 & 3.842 & $\mathbf{2 , 7 6}$ & $\mathbf{5 5 , 5 4}$ & $3,39 \mathrm{E}+06$ & $2,90 \mathrm{E}+07$ \\
\hline 5 & - & 19 & 9 & 59 & 262 & 3.481 & $\mathbf{3 , 1 0}$ & $\mathbf{7 4 , 3 1}$ & $2,26 \mathrm{E}+06$ & $6,17 \mathrm{E}+06$ \\
\hline$\ldots$ & $\ldots$ & $\ldots$ & $\ldots$ & $\ldots$ & $\ldots$ & $\ldots$ & $\mathbf{\ldots}$ & $\ldots$ & $\ldots$ & $\ldots$ \\
\hline 7.000 & - & 19 & 10 & 74 & 261 & 3.484 & $\mathbf{2 , 9 1}$ & $\mathbf{6 1 , 0 7}$ & $2,81 \mathrm{E}+06$ & $1,75 \mathrm{E}+07$ \\
\hline
\end{tabular}




\begin{tabular}{lc}
\hline & CRITÉRIO DE DES \\
\hline & \\
\hline N projeto & $\mathbf{5 . 9 2 E + 0 6}$ \\
\hline Confiabilidade & $\mathbf{5 0 . 1 \%}$ \\
\hline Risco de "falha" & $\mathbf{4 9 . 9 \%}$ \\
\hline & \\
& \\
\hline \multicolumn{2}{c}{ Estatistica Simulação Nf(Et) } \\
\hline mínimo & $3.01 \mathrm{E}+06$ \\
\hline máximo & $1.56 \mathrm{E}+07$ \\
\hline média & $5.92 \mathrm{E}+06$ \\
\hline desvio padrão & $1.32 \mathrm{E}+00$ \\
\hline contagem & 7,000 \\
\hline CV & $2.22 \mathrm{E}-07$ \\
\hline erro $(\%)$ & $0.06 \%$ \\
\hline
\end{tabular}
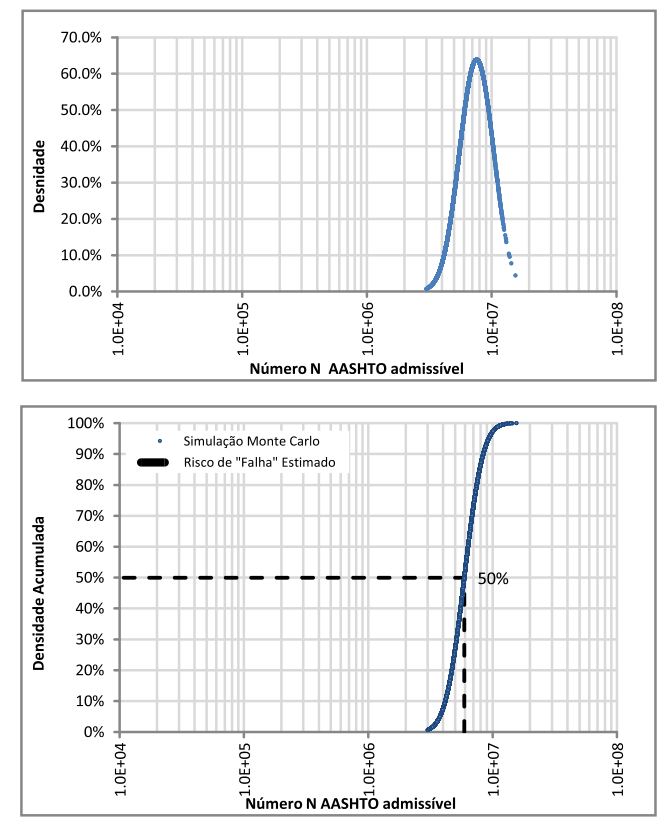

Figura 2. Análise Probabilística e confronto com valores de projeto (critério $\varepsilon_{\mathrm{t}}$ )

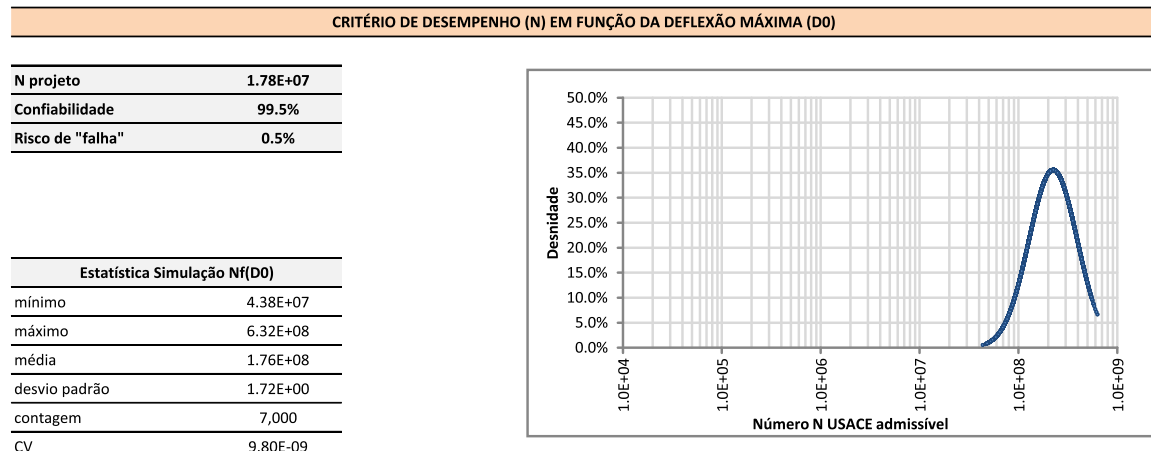

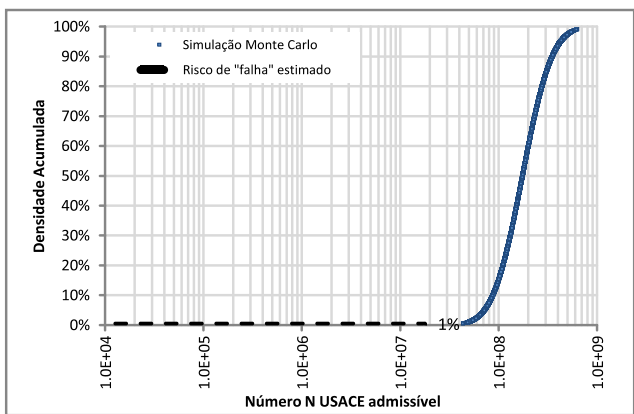

Figura 3. Análise Probabilística e confronto com valores de projeto (critério $D_{0}$ )

\subsection{Etapas $\mathrm{F} \mathrm{e} \mathrm{G}$}

As etapas $\mathrm{F}$ e $\mathrm{G}$ foram sintetizadas através de montagem de gráficos de densidade e densidade acumulada, conforme exemplos apresentados nas Figura 2 e Figura 3.

Tabela 11. Resultados finais das análises

\begin{tabular}{lccc}
\hline Segmento & Critério & $\begin{array}{c}\text { Confiabilidade } \\
\text { Relativa }\end{array}$ & $\begin{array}{c}\text { Risco de } \\
\text { "falha" }\end{array}$ \\
\hline \multirow{2}{*}{ Único } & N AASHTO $\mathrm{f}\left(\varepsilon_{\mathrm{t}}\right)$ & $50,1 \%$ & $49,9 \%$ \\
& N USACE $\left(\mathrm{D}_{0}\right)$ & $99,4 \%$ & $0,6 \%$ \\
\hline
\end{tabular}

O resumo dos resultados obtidos na análise de risco é apresentado na Tabela 11.

A análise realizada através da metodologia proposta indicou que o risco de falha da estrutura frente às hipóteses

de projeto é relativamente alta $(49,9 \%)$. É importante ressaltar que muito embora o risco seja alto, isto não quer dizer que a estrutura irá necessariamente apresentar problemas precoces. Este resultado reflete, em verdade, o que muito acontece quando da aplicação de critérios determinísticos de dimensionamento, tal qual preconizado pelas normas vigentes (DER/SP, DERSA e DNIT). Ou seja, provavelmente foram considerados parâmetros médios fixos na etapa de dimensionamento sem considerar a variação estatística dos parâmetros estruturais, resultando em níveis de confiabilidade relativa ao projeto próximos a $50 \%$.

O resultado da análise apresentada na Tabela 11 também pode ser entendido como um indicador de que a combinação da dispersão dos valores de módulos retroanalisados e da dispersão admitida para os módulos e espessuras das demais camadas pode vir a potencializar os riscos de "falha" do pavimento analisado frente às hipóteses de projeto. 


\subsection{Etapa $\mathrm{H}$ - Embasamento para tomada de ações corretivas}

A etapa $\mathrm{H}$ foi realizada através da regressão dos valores obtidos nas simulações. Com base nos 7.000 casos simulados, foi realizada uma regressão estatística múltipla com auxílio de ferramenta específica do excel®, contemplando-se os valores simulados de módulo e espessuras das camadas e os valores calculados de probabilidade de sucesso da estrutura (confiabilidade relativa ao projeto). A equação obtida neste estudo é apresentada a seguir.

$$
\begin{aligned}
& C=-40,247+2,206 \cdot \log \left(H_{2}\right)+5,226 \cdot \log \left(H_{3}\right) \\
& +1,775 \cdot \log \left(E_{1}\right)+5,569 \cdot \log \left(E_{2}\right)+4,210 \cdot \log \left(E_{3}\right)
\end{aligned}
$$

Onde: $C=$ confiabilidade relativa ao projeto ou probabilidade de sucesso da estrutura; $H_{2}=$ espessura da camada de base $(\mathrm{cm}) ; H_{3}=$ espessura da camada de revestimento $(\mathrm{cm})$; $\mathrm{E}_{1}=$ módulo elástico da camada de subleito ou infraestrutura equivalente $(\mathrm{MPa}) ; E_{2}=$ módulo elástico da camada de base $(\mathrm{MPa}) ; E_{3}=$ módulo elástico da camada de revestimento (MPa). A regressão apresentou valor de $R^{2}=0,96$.

Ressalta-se que a equação (9) tem uso restrito apenas ao caso particular em estudo, não podendo ser aplicada a outras estruturas.

Com base na equação obtida para o caso em estudo, poderiam ser calculadas melhorias nos parâmetros estruturais das camadas de modo a elevar os níveis de confiabilidade relativa global da estrutura para o nível desejado. A Tabela 12 ilustra as alternativas propostas para o estudo.

Tabela 12. Alternativas propostas para elevar nível de

\begin{tabular}{|c|c|c|c|c|c|c|}
\hline Caso & $\begin{array}{c}\mathrm{H}_{2} \\
(\mathrm{~cm}) \\
\end{array}$ & $\begin{array}{c}\mathrm{H}_{3} \\
(\mathrm{~cm}) \\
\end{array}$ & $\begin{array}{c}\mathbf{E}_{1} \\
(\mathrm{MPa})\end{array}$ & $\begin{array}{c}\mathbf{E}_{2} \\
(\mathbf{M P a})\end{array}$ & $\begin{array}{c}\mathbf{E}_{3} \\
(\mathbf{M P a})\end{array}$ & $C$ \\
\hline Situação atual & 20 & 10 & 144 & 300 & 3.750 & $52 \%$ \\
\hline Alternativa 1 & 20 & 10 & 206 & 300 & 3.750 & $80 \%$ \\
\hline Alternativa 2 & 22 & 11 & 144 & 300 & 3.750 & $83 \%$ \\
\hline Alternativa 3 & 20 & 10 & 144 & 350 & 3.750 & $89 \%$ \\
\hline
\end{tabular}
confiabilidade relativa da estrutura

As alternativas apontadas na Tabela 12 são descritas com maiores detalhes a seguir:

- Alternativa 1 - estabilizar as camadas de infraestrutura (subleito / reforço do subleito) de modo a obter um módulo equivalente de $206 \mathrm{MPa}$;

- Alternativa 2 - incrementar os valores de espessuras dos materiais de base e revestimento a serem executados (compensação);

- Alternativa 3 - estabilizar ou substituir o material de base a ser executado de modo a atingir um valor de módulo da ordem de $350 \mathrm{MPa}$ (compensação).

\section{CONCLUSÕES}

O presente trabalho apresentou metodologia de análise de risco, com o objetivo de avaliar estruturas de pavimento recém-projetadas e/ou recém-construídas, visando elevarem-se os níveis de confiabilidade das etapas de projeto e de construção de pavimentos asfálticos flexíveis.

Importante citar que o presente trabalho propõe o controle do módulo da camada do subleito não somente através de correlações com o parâmetro $\mathrm{D}_{0}$ mas também com demais parâmetros da bacia de curvatura $\left(\mathrm{D}_{25}, \mathrm{D}_{120}\right.$, SCI, CF, BDI e BCI). Para a confirmação dos valores de módulo do subleito, bem como para o controle dos parâmetros das demais camadas, recomenda-se a leitura da bacia de deflexões completa com equipamento tipo FWD e realização de procedimentos de retroanálise com uso de softwares específicos para esta aplicação.

Com base na metodologia proposta e nos estudos de caso apresentados, pode-se notar que, em muitos casos, mesmo que grande parte dos pontos ensaiados no controle deflectométrico se apresente abaixo do limite estabelecido em projeto, a análise de risco proposta poderia indicar baixa confiabilidade relativa da estrutura frente às hipóteses de projeto. Isto se dá especialmente quando o pavimento é dimensionado através de métodos determinísticos, tais como preconizam grande parte das normas nacionais vigentes. Em todos os casos, a estabilização do solo do subleito é sempre uma ótima alternativa para diminuir a dispersão de valores de deflexão e, consequentemente, de valores de módulo equivalente da infraestrutura a baixo custo, elevandose o nível de confiabilidade relativa global da estrutura construída frente ao cenário de projeto.

Neste sentido, os autores indicam como prática construtiva preventiva, principalmente em locais com solos de fundação mais heterogêneos, a tomada de medidas preventivas como a escavação do solo da camada final de terraplenagem (CFT) a níveis de cerca de $30 \mathrm{~cm}$ a $60 \mathrm{~cm}$ além da cota original programada para o subleito, com o objetivo de verificarem-se os níveis deflectométricos da infraestrutura do pavimento, antes mesmo de se iniciarem os serviços de melhoria/preparação do subleito.

Desta maneira, caso os níveis deflectométricos da fundação apresentem-se acima dos parâmetros de controle estipulados para o subleito na etapa de projeto ou com elevada dispersão, seria possível, e bastante viável, a estabilização do solo local ou a substituição por outro tipo de material (solos selecionados provenientes de jazidas, materiais reciclados, materiais usinados etc.), visando-se elevar o nível de confiabilidade relativa global da estrutura construída frente às hipóteses admitidas em projeto.

Além disso, outra prática recomendável seria a de se realizar uma análise de risco na etapa de projeto, simulandose as variações que poderiam ocorrer na obra, com respeito ao controle dos valores de deflexão (módulos das camadas) e espessuras das camadas. Com isto, certamente seria possível conceber estruturas com maiores níveis de confiabilidade relativa e menor potencial de "falha" precoce.

Cabe também ressaltar a importância do uso dos índices de curvatura apresentados na Tabela 1 para o estabelecimento de critérios de controle e aceitação de camadas, bem como a importância da coleta e processamento destas informações para fins de retroanálise dos valores de módulo das camadas. O uso destes indicadores levará a uma interpretação mais assertiva do comportamento estrutural dos pavimentos, especialmente para fins de aplicação da metodologia proposta a partir de dados exclusivos de controle de campo.

Em termos gerais, pode-se afirmar que a metodologia de controle proposta vem ao encontro das necessidades verificadas nas etapas de projeto e construção e apresentou boa aplicabilidade segundo os estudos de caso realizados no item 3 do presente trabalho. A metodologia poderá fornecer 
os passos principais para um controle de qualidade efetivo e para a tomada de decisões bem fundamentadas.

\section{REFERÊNCIAS}

\section{AMERICAN ASSOCIATION OF STATE HIGHWAY AND TRANSPORTATION OFFICIALS. AASHTO Guide for Pavement Structures. AASHTO, ISBN: 1-56051-055-2. Washington, D.C., 1993.}

ANDREATINI, L. F. V. Sugestões para o estabelecimento de sistemas de controle estatístico específicos para a área de pavimentação. In: 23 Reunião Anual de Pavimentação. Florianópolis, S.C., 1988.

APEAGYEI, ALEX K.; HOSSAIN, M. SHABBIR. Evaluation of the Lightweight Deflectometer for In-Situ Determination of Pavement Layer Moduli. Virginia Transportation Research Council. Virginia, 2010.

DEPARTAMENTO DE ESTRADAS DE RODAGEM DE SÃO PAULO. DER/SP IP-DE-P00/001: INSTRUÇÃO DE PROJETO DE PAVIMENTAÇÃO. São Paulo, 2006.

DEPARTAMENTO NACIONAL DE ESTRADAS DE RODAGEM. DNER-ME 024/94: Determinação de Deflexões pela Viga Benkelman. Rio de Janeiro, 1994.

DEPARTAMENTO NACIONAL DE INFRAESTRUTURA DE TRANSPORTES. DNIT 013/2004 - PRO Requisitos para a qualidade na execução de obras rodoviárias - Procedimento. Rio de Janeiro, 2004.

DNIT 133/2010-ME: Pavimentação asfáltica Delineamento da linha de influência longitudinal da bacia de deformação por intermédio da Viga Benkelman. Rio de Janeiro, 2010 .

Manual de Pavimentação. Publicação IPR 719. Rio de Janeiro, 2006

FERRI, S. Critérios de aceitação e controle da qualidade da execução de camadas de fundação de pavimentos novos através de métodos deflectométricos. Dissertação (Mestrado). Escola Politécnica da Universidade de São Paulo, São Paulo, 2013.

GHARAIBEH, NASIR G.; GARBER, SABRINA I.; LIU

LITAO. Determining Optimum Sample Size for Percent-WithinLimits Specifications. Transportation Research Record: Journal of the Transportation Research Board, No. 2151, Transportation Research Board of the National Academies, Washington, D.C., 2010. DOI: $10.3141 / 2151-10$.

LOPES, F. M.; SANTOS, C. R. G.; FORTES, R. M.; FERRI, S.; SUZUKI, C. Y. Proposta de Metodologia para avaliação e pavimentos asfálticos utilizando características da bacia deflectométrica. In: $4^{\circ}$ Congresso de Infraestrutura de Transportes - Coninfra 2010. São Paulo, SP, 2010. ISSN 19833903.

MCCABE B., S. AbouRizk; GAVIN, J. Sample Size Analysis for Asphalt Pavement Quality Control. Journal of Infrastructure Systems. Vol. 5, No. 4, 1999.

MOTTA, L. M. G. \& MEDINA, J. Mecânica dos Pavimentos. 2a Edição, Rio de Janeiro, 2005.

NAZZAL, M. D.; MOHAMMAD L. N. (2010). Estimation of Resilient Modulus of Subgrade Soils Using Falling Weight Deflectometer. Transportation Research Record: Journal of the
Transportation Research Board, No. 2186, Transportation Research Board of the National Academies, Washington, D.C., 2010, pp. 1-10.

PATHOMVANICH, S.; NAJAFI, F. T.; KOPAC, P. A. Procedure for Monitoring and Improving Effectiveness of Quality Assurance Specifications. Journal Transportation Research Record: Journal of the Transportation Research Board, 2002. ISSN 0361-1981.

PINTO, S. \& PREUSSLER, E. Pavimentação rodoviária: conceitos fundamentais sobre pavimentos flexíveis. Editora Copiarte, ISBN: 85-902537-1-6. Rio de Janeiro, Brasil, 2002.

PREFEITURA MUNICIPAL DE SÃO PAULO / SECRETARIA DE INFRAESTRUTURA URBANA E OBRAS. PMSP/SIURB IP-08: Instrução de Projeto para Análise Mecanicista à Fadiga de Estruturas de Pavimento. São Paulo, 2004.

SANTOS, C. R. G. Dimensionamento e análise do ciclo de vida de pavimentos rodoviários: uma abordagem probabilística. Tese (Doutorado). Escola Politécnica da Universidade de São Paulo, São Paulo, 2011.

TRANSPORTATION RESEARCH BOARD NCHRP NATIONAL COOPERATIVE HIGHWAY RESEARCH PROGRAM. NCHRP REPORT 626: NDT Technology for Quality Assurance of HMA Pavement Construction. Project 1065. ISSN 0077-5614. ISBN: 978-0-309-11777-7. Washington, D.C., 2009.

VOLOVSKI, M.; LABI, S.; SOMMER, K.; NOURELDIN, S; WALKER, R. Developing Statistical Limits for Using the Light Weight Deflectometer (LWD) in Construction Quality Assurance. In: ANNUAL MEETING OF TRANSPORTANTION RESEARCH BOARD, 95., 2016, Washington D.C. Proceedings... Washington D.C.: The National Academies, 2016. 